\title{
Stability and Bifurcation of a Class of Discrete-Time Cohen-Grossberg Neural Networks with Delays
}

\author{
Qiming Liu, Rui Xu, and Zhiping Wang \\ Institute of Applied Mathematics, Shijiazhuang Mechanical Engineering College, \\ Shijiazhuang 050003, China \\ Correspondence should be addressed to Qiming Liu, lqmmath@yahoo.com.cn
}

Received 5 November 2010; Accepted 3 January 2011

Academic Editor: Rigoberto Medina

Copyright (C) 2011 Qiming Liu et al. This is an open access article distributed under the Creative Commons Attribution License, which permits unrestricted use, distribution, and reproduction in any medium, provided the original work is properly cited.

\begin{abstract}
A class of discrete-time Cohen-Grossberg neural networks with delays is investigated in this paper. By analyzing the corresponding characteristic equations, the asymptotical stability of the null solution and the existence of Neimark-Sacker bifurcations are discussed. By applying the normal form theory and the center manifold theorem, the direction of the Neimark-Sacker bifurcation and the stability of bifurcating periodic solutions are obtained. Numerical simulations are given to illustrate the obtained results.
\end{abstract}

\section{Introduction}

Hopfield [1] proposed Hopfield neural networks in 1982, and in 1983, Cohen and Grossberg [2] proposed Cohen-Grossberg neural networks, which include Hopfield neural networks. These network models have been successfully applied to signal processing, pattern recognition, optimization, and associative memories. The analysis of the dynamical behaviors is a necessary step for practical design of neural networks because its applications heavily depend on the dynamical behaviors, and considerable work has been done to develop the dynamics such as stability and periodicity [3-14]. In order to obtain a deep and clear understanding of the dynamics of complicated neural networks with time delays, researchers have focused on the studying of simple systems. This is indeed very useful since the complexity found may be carried over to large networks.

In applications of continuous-time neural networks with or without delays to some practical problems, such as computer simulation, experimental, or computational purposes, it is usual to formulate a discrete-time system which is a discrete version of the continuoustime system, while their discrete-time counterparts have only been in the spotlight since 2000 . 
Bifurcation analysis for some models has been undertaken [15-22]; the stability of the equilibrium and Neimark-Sacker bifurcation for a Cohen-Grossberg system without delays are discussed in [15]. To the best of the author's knowledge, no similar results have been obtained for discrete-time Cohen-Grossberg system with delays.

The objective of this paper is to study the following two-neuron discrete-time CohenGrossberg neural networks with discrete delays:

$$
\begin{aligned}
& x_{1}(n+1)=x_{1}(n)-a_{1}\left(x_{1}(n)\right)\left[b_{1}\left(x_{1}(n)\right)-d_{1} f_{2}\left(x_{2}\left(n-k_{2}\right)\right)\right] \\
& x_{2}(n+1)=x_{2}(n)-a_{2}\left(x_{2}(n)\right)\left[b_{2}\left(x_{2}(n)\right)-d_{2} f_{1}\left(x_{1}\left(n-k_{1}\right)\right)\right]
\end{aligned}
$$

where $x_{i}(n)$ denote the state variable of the $i$ th neuron; $a_{i}(\cdot)$ represent amplification functions which are positive for $R ; f_{i}(\cdot)$ denote the signal functions of the $i$ th neuron; $b_{i}(\cdot)$ are appropriately behaved functions; $d_{i}(\cdot)$ are connection weights of the neural networks; discrete delays $k_{i}$ correspond to the finite speed of the axonal signal transmission, $i=1,2$.

The rest of this paper is organized as follows: the asymptotical stability and bifurcation are analyzed for the system in Section 2. Based on the normal form method and the center manifold theorem, the formula for determining the direction of Neimark-Sacker bifurcation, stability of bifuricating periodic solutions of the model are derived in Section 3. An example is given in Section 4 to demonstrate the main results. Conclusions are finally drawn in Section 5.

\section{Stability Analysis and Existence of Bifurcations}

Throughout this paper, we assume that

$$
\left(\mathrm{H}_{1}\right) b_{1}(0)=b_{2}(0)=0, f_{1}(0)=f_{2}(0)=0 \text {; }
$$

$\left(\mathrm{H}_{2}\right) 0<a_{i}(0) b_{i}^{\prime}(0)<1, i=1,2$. system:

Let $u_{1}(n)=x_{1}\left(n-k_{1}\right), u_{2}(n)=x_{2}(n)$; we transform system (1.1) into the following

$$
\begin{gathered}
u_{1}(n+1)=u_{1}(n)-a_{1}\left(u_{1}(n)\right)\left[b_{1}\left(u_{1}(n)\right)-d_{1} f_{2}\left(u_{2}(n-k)\right)\right], \\
u_{2}(n+1)=u_{2}(n)-a_{2}\left(u_{2}(n)\right)\left[b_{2}\left(u_{2}(n)\right)-d_{2} f_{1}\left(u_{1}(n)\right)\right]
\end{gathered}
$$

where $k=k_{1}+k_{2}$.

Furthermore, system (2.1) can be transformed into the following system of $k+2$ difference equations without delays:

$$
\begin{gathered}
u_{1}^{(0)}(n+1)=u_{1}^{(0)}(n)-a_{1}\left(u_{1}^{(0)}(n)\right)\left[b_{1}\left(u_{1}^{(0)}(n)\right)-d_{1} f_{2}\left(u_{2}^{(k)}(n)\right)\right], \\
u_{2}^{(0)}(n+1)=u_{2}^{(0)}(n)-a_{2}\left(u_{2}^{(0)}(n)\right)\left[b_{2}\left(u_{2}^{(0)}(n)\right)-d_{2} f_{1}\left(u_{1}^{(0)}(n)\right)\right], \\
u_{2}^{(j)}(n+1)=u_{2}^{(j-1)}(n), \quad j=1,2, \ldots, k .
\end{gathered}
$$


In the following discussion, for convenience, we denote

$$
\begin{gathered}
b=a_{1}(0) a_{2}(0) d_{1} d_{2} f_{1}^{\prime}(0) f_{2}^{\prime}(0), \quad \alpha_{i}=1-a_{i}(0) b_{i}^{\prime}(0), \\
a_{i}=a_{i}(0), \quad a_{i}^{\prime}=a_{i}^{\prime}(0), \quad a_{i}^{\prime \prime}=a_{i}^{\prime \prime}(0), \quad b_{i}=b_{i}(0), \quad b_{i}^{\prime}=b_{i}^{\prime}(0), \quad b_{i}^{\prime \prime}=b_{i}^{\prime \prime}(0), \quad b_{i}^{\prime \prime \prime}=b_{i}^{\prime \prime \prime}(0), \\
f_{i}=f_{i}(0), \quad f_{i}^{\prime}=f_{i}^{\prime}(0), \quad f_{i}^{\prime \prime}=f_{i}^{\prime \prime}(0), \quad f_{i}^{\prime \prime \prime}=f_{i}^{\prime \prime \prime}(0)
\end{gathered}
$$

for $i=1,2$.

The Jacobian matrix of system $(2.2)$ at the fixed point $(0, \ldots, 0)$ is defined as follows:

$$
\widehat{A}=\left(\begin{array}{cccccc}
\alpha_{1} & 0 & 0 & \cdots & 0 & a_{1} d_{1} f_{2}^{\prime} \\
a_{2} d_{2} f_{1}^{\prime} & \alpha_{2} & 0 & \cdots & 0 & 0 \\
0 & 1 & 0 & \cdots & 0 & 0 \\
0 & 0 & 1 & \cdots & 0 & 0 \\
\vdots & \vdots & \vdots & \cdots & \vdots & \vdots \\
0 & 0 & 0 & \cdots & 1 & 0
\end{array}\right) .
$$

The associated characteristic equation of system (2.2) is

$$
\left(\lambda-\alpha_{1}\right)\left(\lambda-\alpha_{2}\right) \lambda^{k}=b
$$

Suppose that $\lambda=e^{i \theta}$ is a root of the characteristic equation and substitute $e^{i \theta}$ into (2.5); then we have

$$
\left(\cos \theta-\alpha_{1}+i \sin \theta\right)\left(\cos \theta-\alpha_{2}+i \sin \theta\right)(\cos k \theta+i \sin k \theta)=b .
$$

When $\theta \in(0, \pi)$, separating the real and imaginary parts of (2.6), we have

$$
\begin{gathered}
b=\sqrt{\left(1+\alpha_{1}^{2}-2 \alpha_{1} \cos \theta\right)\left(1+\alpha_{2}^{2}-2 \alpha_{2} \cos \theta\right)} \cosh (\theta), \\
\sin h(\theta)=0
\end{gathered}
$$

where

$$
h(\theta)=\cot ^{-1}\left(\frac{\cos \theta-\alpha_{1}}{\sin \theta}\right)+\cot ^{-1}\left(\frac{\cos \theta-\alpha_{2}}{\sin \theta}\right)+k \theta
$$


in which $\cot ^{-1}$ denotes the inverse of the cotangent function restricted to the interval $(0, \pi)$. Since

$$
\begin{aligned}
h^{\prime}(\theta)= & \frac{1}{1+\left(\left(\cos \theta-\alpha_{1}\right) / \sin \theta\right)^{2}} \times \frac{1-\alpha_{1} \cos \theta}{\sin ^{2} \theta} \\
& +\frac{1}{1+\left(\left(\cos \theta-\alpha_{2}\right) / \sin \theta\right)^{2}} \times \frac{1-\alpha_{2} \cos \theta}{\sin ^{2} \theta}+k>0
\end{aligned}
$$

for $\theta \in(0, \pi)$ and

$$
\lim _{\theta \rightarrow \pi^{-}} h(\theta)=(k+2) \pi, \quad \lim _{\theta \rightarrow 0^{+}} h(\theta)=0,
$$

we know that $h(\theta):(0, \pi) \rightarrow(0,(k+2) \pi)$ is an increasing bijective function.

We know from the second equation in $(2.7)$ that $h(\theta)=j \pi$. Denote $\theta_{j}=h^{-1}(j \pi), j=$ $1,2, \ldots,(k+1)$; then we have from the first equation in $(2.7)$ that

$$
b=b_{j}=(-1)^{j} \sqrt{\left(1+\alpha_{1}^{2}-2 \alpha_{1} \cos \theta_{j}\right)\left(1+\alpha_{2}^{2}-2 \alpha_{2} \cos \theta_{j}\right)}, \quad j=1,2, \ldots, k+1 .
$$

When $\theta=0, b=b_{0}=\left(1-\alpha_{1}\right)\left(1-\alpha_{2}\right)>0$.

When $\theta=\pi, b=b_{k+2}=(-1)^{k+2}\left(1+\alpha_{1}\right)\left(1+\alpha_{2}\right)$.

Obviously, if $\theta$ is a root of (2.6), $-\theta$ is also the root of (2.6). So, we only need to consider the characteristic roots $e^{i \theta}$ of $(2.5)$ in $[0, \pi]$. Furthermore, from the value $b_{j}$ given above, we have

$$
\cdots<b_{5}<b_{3}<b_{1}<0<b_{0}<b_{2}<b_{4} \cdots
$$

On the other hand, we have from (2.5) that

$$
\frac{d|\lambda|^{2}}{d b}=\frac{d \lambda \bar{\lambda}}{d b}=\lambda \frac{d \bar{\lambda}}{d b}+\bar{\lambda} \frac{d \lambda}{d b}=\frac{2 \operatorname{Re}(\lambda P(\lambda))}{|P(\lambda)|^{2}}
$$

where $P(\lambda)=\lambda^{k-1}\left[\lambda\left(\lambda-\alpha_{1}\right)+\lambda\left(\lambda-\alpha_{2}\right)+k\left(\lambda-\alpha_{1}\right)\left(\lambda-\alpha_{2}\right)\right]$.

It follows that

$$
\left.\frac{d|\lambda|^{2}}{d b}\right|_{b=b_{j}}=b_{j} \frac{1}{\left|P\left(e^{i \theta_{j}}\right)\right|^{2}}\left(k+\frac{1-\alpha_{1} \cos \theta_{j}}{1+\alpha_{1}^{2}-2 \alpha_{1} \cos \theta_{j}}+\frac{1-\alpha_{2} \cos \theta_{j}}{1+\alpha_{2}^{2}-2 \alpha_{2} \cos \theta_{j}}\right) .
$$

Hence we have

$$
\operatorname{sign}\left\{\left.\frac{d|\lambda|^{2}}{d b}\right|_{b=b_{j}}\right\}=\operatorname{sign}\left\{b_{j}\right\}=(-1)^{j}
$$


Since the roots of the characteristic equations (2.5) are $\alpha_{1}, \alpha_{2}$, and 0 when $b=0$, they are obviously inside the unit circle, so the null solution of system (2.2) is asymptotically stable. As the parameter $b$ varies, the number of roots of the characteristic equation out of the circle can change if a root appears on or crosses the unit circle. According to (2.12) and (2.15), we obtain that the null solution of system (2.2) is asymptotically stable if and only if $b \in\left(b_{1}, b_{0}\right)$.

At the critical points $b=b_{0}$ and $b=b_{1}$, bifurcation phenomena occur.

When $b=b_{0}=\left(1-\alpha_{1}\right)\left(1-\alpha_{2}\right)$, the characteristic equation of system (2.2) has a simple root $\lambda=1$ on the unit circle, and all the other roots are inside the unit circle. Therefore, a Fold bifurcation occurs at the origin in system $(2.2)([20,21])$.

When $b=b_{1}$, the characteristic equation of system (2.2) has a simple pair of roots $e^{ \pm i \theta_{1}}$ on the unit circle, and all the other roots are inside the unit circle. Since $\theta_{1}=h^{-1}(\pi)$, we have that $\theta_{1}$ satisfies the following equation:

$$
\pi=\cot ^{-1}\left(\frac{\cos \theta-\alpha_{1}}{\sin \theta}\right)+\cot ^{-1}\left(\frac{\cos \theta-\alpha_{2}}{\sin \theta}\right)+k \theta .
$$

It follows from (2.16) that

$$
-\cot k \theta=\cot \left(\cot ^{-1}\left(\frac{\cos \theta-\alpha_{1}}{\sin \theta}\right)+\cot ^{-1}\left(\frac{\cos \theta-\alpha_{2}}{\sin \theta}\right)\right)
$$

which leads to

$$
\sin (k+2) \theta-\left(\alpha_{1}+\alpha_{2}\right) \sin (k+1) \theta+\alpha_{1} \alpha_{2} \sin k \theta=0 .
$$

So, $\theta_{1}$ satisfies (2.16) if and only if it satisfies (2.18).

Let

$$
y_{1}=\sin (k+2) \theta, \quad y_{2}=\left(\alpha_{1}+\alpha_{2}\right) \sin (k+1) \theta-\alpha_{1} \alpha_{2} \sin k \theta,
$$

$y_{1}$ and $y_{2}$ are easy to be proved to be convex function; furthermore, $y_{1}(0)=y_{2}(0)=0, y_{1}^{\prime}\left(0^{+}\right)>$ $y_{2}^{\prime}\left(0^{+}\right)$, and $y_{1}(\pi /(k+2))=0<y_{2}(\pi /(k+2))$; then curves $y_{1}$ and $y_{2}$ have a unique intersection in positive quadrant for $\theta$ in $(0, \pi /(k+2))$, that is, (2.18) has a unique solution $\theta=\theta_{1}$ in $(0, \pi /(k+2))$. Then

$$
b_{1}=-\sqrt{\left(1+\alpha_{1}^{2}-2 \alpha_{1} \cos \theta_{1}\right)\left(1+\alpha_{2}^{2}-2 \alpha_{2} \cos \theta_{1}\right)} .
$$

As $\theta_{1} \in(0, \pi /(k+2))$, so $e^{ \pm i \theta_{1}} \neq 1, e^{ \pm i \theta_{1}}$ is not a root of order $1,2,3$, or 4 of the unity. We also know from $(2.15)$ that $\left.(d|\lambda| / d b)\right|_{b=b_{1}} \neq 0$. Hence, a Neimark-Sacker bifurcation occurs at the origin in system (2.2) at $b=b_{1}$ [23].

From the discussions above, we have the following results. 
Theorem 2.1. Under assumptions $\left(H_{1}\right)-\left(H_{2}\right)$, we have that

(1) if $b \in\left(b_{1}, b_{0}\right)$, the null solution of system (1.1) is asymptotically stable,

(2) if $b=b_{0}$, a Fold bifurcation occurs at the origin in system (1.1),

(3) if $b=b_{1}$, a Neimark-Sacker bifurcation occurs at the origin in system (1.1), that is, a unique closed invariant curve bifurcates from the origin near $b=b_{1}$,

where

$$
b_{0}=\left(1-\alpha_{1}\right)\left(1-\alpha_{2}\right), \quad b_{1}=-\sqrt{\left(1+\alpha_{1}^{2}-2 \alpha_{1} \cos \theta_{1}\right)\left(1+\alpha_{2}^{2}-2 \alpha_{2} \cos \theta_{1}\right)}
$$

in which $\theta_{1}$ is the unique solution in $(0, \pi /(k+2))$ of the equation $\sin (k+2) \theta-\left(\alpha_{1}+\alpha_{2}\right) \sin (k+$ 1) $\theta+\alpha_{1} \alpha_{2} \sin k \theta=0$.

Remark 2.2. Similar to the case when $b=b_{1}$, if $b=b_{j}, j=2,3, \ldots, k+1$, a Neimark-Sacker bifurcation also occurs at the origin in system (1.1) near $b=b_{j}$ except $\theta_{j}=2 \pi / 3$ and $\pi / 2$. If $b=b_{k+2}=(-1)^{k}\left(1+\alpha_{1}\right)\left(1+\alpha_{2}\right)$, the characteristic equation has a simple root $\lambda=-1$ on the unit circle, and a flip bifurcation occurs in system (1.1). If $|b|>\left|b_{k+2}\right|=\left(1+\alpha_{1}\right)\left(1+\alpha_{2}\right)$, all roots of the characteristic equation are outside the unit circle, and system (1.1) may be chaostic (as shown in Figures 4 and 5).

Furthermore, under some conditions which have more restrictions than $\left(\mathrm{H}_{2}\right)$ and $b \in$ $\left(-b_{1}, b_{0}\right)$, the null solution of system (1.1) is globally asymptotically stable.

Theorem 2.3. Under assumption $\left(H_{1}\right)$, the null solution of system (1) is globally asymptotically stable if the following conditions hold.

$\left(\mathrm{H}_{3}\right)$ There exist constants $\underline{a}_{i}$ and $\bar{a}_{i}$ such that $0<\underline{a}_{i} \leq a_{i}(\cdot) \leq \bar{a}_{i}$ for $i=1,2$.

$\left(\mathrm{H}_{4}\right)$ There exist constants $\underline{b}_{i}$ and $L_{i}$ such that $b_{i}^{\prime}(\cdot) \geq \underline{b}_{i}>0$ and $\left|f_{i}^{\prime}(\cdot)\right| \leq L_{i}$ for $i=1,2$.

$\left(\mathrm{H}_{5}\right) 0<\underline{a}_{i} \underline{b}_{i}<1$ for $i=1,2$, and $\bar{a}_{1} \bar{a}_{2}\left|d_{1}\right|\left|d_{2}\right| L_{1} L_{2} \leq \underline{a}_{1} \underline{a}_{2} \underline{b}_{1} \underline{b}_{2}$.

Proof. Since $\bar{a}_{1} \bar{a}_{2} d_{1} d_{2} L_{1} L_{2} \leq \underline{a}_{1} \underline{a}_{2} \underline{b}_{1} \underline{b}_{2}$, the following matrix

$$
A=\left(\begin{array}{cc}
\underline{a}_{1} \underline{b}_{1} & -\bar{a}_{1}\left|d_{1}\right| L_{2} \\
-\bar{a}_{2}\left|d_{2}\right| L_{1} & \underline{a}_{2} \underline{b}_{2}
\end{array}\right),
$$

is an $M$-matrix, and there exists a vector $p=\left(p_{i}\right)_{1 \times 2}>0$ such that $p A>0([24])$, that is,

$$
p_{1} \underline{a}_{1} \underline{b}_{1}-p_{2} \bar{a}_{2}\left|d_{2}\right| L_{1}>0, \quad p_{2} \underline{a}_{2} \underline{b}_{2}-p_{1} \bar{a}_{1}\left|d_{1}\right| L_{2}>0 .
$$

Hence, we can choose a constant $\lambda>1$ such that

$$
p_{1}\left(1-\lambda+\underline{a}_{1} \underline{b}_{1} \lambda\right)-p_{2} \bar{a}_{2}\left|d_{2}\right| L_{1} \lambda>0, \quad p_{2}\left(1-\lambda+\underline{a}_{2} \underline{b}_{2} \lambda\right)-p_{1} \bar{a}_{1}\left|d_{1}\right| L_{2} \lambda^{k+1}>0 .
$$


Let $U_{1}(n)=\lambda^{n}\left|u_{1}(n)\right|, U_{2}(n)=\lambda^{n}\left|u_{2}(n)\right|$; then we have from (1.1) that

$$
\begin{gathered}
U_{1}(n)=\lambda^{n+1}\left|u_{1}(n+1)\right| \leq \lambda\left(1-\underline{a}_{1} \underline{b}_{1}\right)\left|U_{1}(n)\right|+\bar{a}_{1}\left|d_{1}\right| L_{2} \lambda^{k+1}\left|U_{2}(n-k)\right|, \\
U_{2}(n)=\lambda^{n+1}\left|u_{2}(n+1)\right| \leq \lambda\left(1-\underline{a}_{2} \underline{b}_{2}\right)\left|U_{2}(n)\right|+\bar{a}_{2}\left|d_{2}\right| L_{1} \lambda\left|U_{1}(n)\right| .
\end{gathered}
$$

Define a Lypunov function $V(t)$ by

$$
V(t)=\sum_{i=1}^{2} p_{i} U_{i}(n)+p_{1} \bar{a}_{1}\left|d_{1}\right| L_{2} \lambda^{k+1} \sum_{l=n-k}^{n-1}\left|U_{2}(l)\right| .
$$

Then we obtain from (2.24) and (2.25) that

$$
\begin{aligned}
\Delta V(n)=V(n+1)-V(n) \leq & -\left[p_{1}\left(1-\lambda+\underline{a}_{1} \underline{b}_{1} \lambda\right)-p_{2} \bar{a}_{2}\left|d_{2}\right| L_{1} \lambda\right] U_{1}(n) \\
& -\left[p_{2}\left(1-\lambda+\underline{a}_{2} \underline{b}_{2} \lambda\right)-p_{1} \bar{a}_{1}\left|d_{1}\right| L_{2} \lambda^{k+1}\right] U_{2}(n) \\
\leq & 0,
\end{aligned}
$$

which implies $V(t) \leq V(0)$.

Note that

$$
\begin{gathered}
V(t) \geq \lambda^{n} m_{0}\left(\left|u_{1}(t)\right|+\left|u_{2}(t)\right|\right) \\
V(0)=\sum_{i=1}^{2} p_{i} U_{i}(0)+p_{1} \bar{a}_{1}\left|d_{1}\right| L_{2} \lambda^{k+1} \sum_{l=-k}^{-1}\left|U_{2}(l)\right| \stackrel{\text { def }}{=} M_{0}
\end{gathered}
$$

where $m_{0}=\min \left\{1 / \bar{a}_{1}, 1 / \bar{a}_{2}\right\}$ and $M_{0}$ is a positive constant.

Thus

$$
\left|u_{1}(t)\right|+\left|u_{2}(t)\right| \leq M_{0} / m_{0}\left(\frac{1}{\lambda}\right)^{n}
$$

where $\lambda>1$. Therefore, $\lim _{n \rightarrow+\infty} u_{i}(t) \rightarrow 0, i=1,2$, that is, the null solution of system (1.1) is globally attractive. Note that $b_{1} \leq-b_{0}$, which implies that $b \in\left(-b_{1}, b_{0}\right)$ if $|b| \leq b_{0}$. It is easy to know that if condition $\left(\mathrm{H}_{4}\right)$ holds, then $\left(\mathrm{H}_{2}\right)$ and $|b| \leq b_{0}$ hold, consequently, the null solution of system (1.1) is asymptotically stable. Then the null solution of system (1.1) is globally asymptotically stable.

Remark 2.4. The conditions under which the null solution is globally asymptotically stable in Theorem 2.3 are less restrictive than ones in Theorem 2.3 in [14] for discrete-time CohenGrossberg system while they are more restrictive than ones for the corresponding continuous Cohen-Grossberg system because $\left|d_{1}\right|\left|d_{2}\right| L_{1} L_{2} \leq \underline{b}_{1} \underline{b}_{2}$ implies the globally asymptotical stability of zero solution for the corresponding continuous system according to Corollary 2 in [8]. 


\section{Direction and Stability of Neimark-Sacker Bifurcation for the Model}

In Section 2, we obtain some conditions under which system (1.1) undergoes Neimark-Sacker bifurcation at the origin. In this section, we investigate the properties of the Neimark-Sacker bifurcation by applying the normal form theory and the center manifold theorem for discrete time system developed by Kuznetsov [23], namely, to determine the direction of NeimarkSacker bifurcation and the stability of periodic solutions bifurcating from the origin of system (1.1).

We still discuss system (2.2). From the discussions in Section 2, we know that if $b=b_{1}$, system (2.2) undergoes a Neimark-Sacker bifurcation at the origin and the associated characteristic equation of system (2.2) has a pair simple imaginary roots $e^{ \pm i \theta_{1}}$.

Denote $\lambda_{1}=e^{i \theta_{1}}$. Let $q \in C^{k+2}$ be an eigenvector of $\widehat{A}$ corresponding to eigenvalue $\lambda_{1}$. Then $\widehat{A} q=\lambda q$. Again, let $p \in C^{k+2}$ be an eigenvector of $\widehat{A}^{T}$ corresponding to its eigenvalue $\bar{\lambda}_{1}$.

By direct calculation, we obtain

$$
\begin{gathered}
q=\left(\frac{a_{1} d_{1} f_{2}^{\prime}}{\lambda_{1}-\alpha_{1}}, \lambda_{1}^{k}, \lambda_{1}^{k-1}, \ldots, \lambda_{1}, 1\right)^{T}, \\
p=E_{1}\left(\frac{\bar{\lambda}_{1}}{a_{1} d_{1} f_{2}^{\prime}}, \frac{1}{\bar{\lambda}_{1}^{k-1}\left(\bar{\lambda}_{1}-\alpha_{2}\right)}, \frac{1}{\bar{\lambda}_{1}^{k-1}}, \frac{1}{\bar{\lambda}_{1}^{k-2}}, \ldots, \frac{1}{\bar{\lambda}_{1}}, 1\right)^{T},
\end{gathered}
$$

in which

$$
E_{1}=\frac{1}{k+2+\left(\alpha_{1} /\left(\lambda_{1}-\alpha_{1}\right)\right)+\left(\alpha_{2} /\left(\lambda_{1}-\alpha_{2}\right)\right)},
$$

where $p$ and $q$ satisfy $\langle p, q\rangle=1$.

System (2.2) can be written as

$$
u(n+1)=\widehat{A} u(n)+F(u(n))
$$

where $u(n)=\left(u_{1}^{(0)}(n), u_{2}^{(0)}(n), \ldots, u_{2}^{(k)}(n)\right)^{T}, F(u(n))=\left(F_{1}(u(n)), F_{2}(u(n)), 0, \ldots, 0\right)^{T}$ in which $F_{i}(i=1,2)$ can be expanded in the form

$$
\begin{aligned}
F_{1}(\xi)= & -\left(a_{1} \frac{b_{1}^{\prime \prime}}{2}+a_{1}^{\prime} b_{1}^{\prime}\right) \xi_{1}^{2}+a_{1} d_{1} \frac{f_{2}^{\prime \prime}}{2} \xi_{k+2}^{2}+a_{1}^{\prime} d_{1} f_{2}^{\prime} \xi_{1} \xi_{k+2} \\
& -\left(a_{1} \frac{b_{1}^{\prime \prime \prime}}{6}+a_{1}^{\prime} \frac{b_{1}^{\prime \prime}}{2}+\frac{a_{1}^{\prime \prime}}{2} b_{1}^{\prime}\right) \xi_{1}^{3}+a_{1} d_{1} \frac{f_{2}^{\prime \prime \prime}}{6} \xi_{k+2}^{3} \\
& +a_{1}^{\prime} d_{1} \frac{f_{2}^{\prime \prime}}{2} \xi_{1} \xi_{k+2}^{2}+\frac{a_{1}^{\prime \prime}}{2} d_{1} f_{2}^{\prime} \xi_{1}^{2} \xi_{k+2}+\text { h.o.t., }
\end{aligned}
$$


Discrete Dynamics in Nature and Society

$$
\begin{aligned}
F_{2}(\xi)= & -\left(a_{2} \frac{b_{2}^{\prime \prime}}{2}+a_{2}^{\prime} b_{2}^{\prime}\right) \xi_{2}^{2}+a_{2} d_{2} \frac{f_{1}^{\prime \prime}}{2} \xi_{1}^{2}+a_{2}^{\prime} d_{2} f_{1}^{\prime} \xi_{1} \xi_{2} \\
& -\left(a_{2} \frac{b_{2}^{\prime \prime \prime}}{6}+a_{2}^{\prime} \frac{b_{2}^{\prime \prime}}{2}+\frac{a_{2}^{\prime \prime}}{2} b_{2}^{\prime}\right) \xi_{2}^{3}+a_{2} d_{2} \frac{f_{1}^{\prime \prime \prime}}{6} \xi_{1}^{3} \\
& +a_{2}^{\prime} d_{2} \frac{f_{1}^{\prime \prime}}{2} \xi_{2} \xi_{1}^{2}+\frac{a_{2}^{\prime \prime}}{2} d_{2} f_{1}^{\prime} \xi_{1} \xi_{2}^{2}+\text { h.o.t.. }
\end{aligned}
$$

and $\widehat{A}$ is defined by (2.4).

It follows that

$$
\begin{aligned}
B_{1}(x, y)= & \left.\sum_{j, k}^{n} \frac{\partial^{2} F_{1}(\xi)}{\partial \xi_{j} \partial \xi_{k}}\right|_{\xi=0} x_{j} y_{k} \\
= & -\left(a_{1} b_{1}^{\prime \prime}+2 a_{1}^{\prime} b_{1}^{\prime}\right) x_{1} y_{1}+a_{1} d_{1} f_{2}^{\prime \prime} x_{k+2} y_{k+2}+a_{1}^{\prime} d_{1} f_{2}^{\prime} x_{1} y_{k+2}, \\
B_{2}(x, y)= & \left.\sum_{j, k}^{n} \frac{\partial^{2} F_{2}(\xi)}{\partial \xi_{j} \partial \xi_{k}}\right|_{\xi=0} x_{j} y_{k} \\
= & -\left(a_{2} b_{2}^{\prime \prime}+2 a_{2}^{\prime} b_{2}^{\prime}\right) x_{2} y_{2}+a_{2} d_{2} f_{1}^{\prime \prime} x_{1} y_{1}+a_{2}^{\prime \prime} d_{2} f_{1}^{\prime} x_{1} y_{2}, \\
C_{1}(x, y, z)= & \left.\sum_{j, k, l}^{n} \frac{\partial^{3} F_{1}(\xi)}{\partial \xi_{j} \partial \xi_{k} \partial \xi_{l}}\right|_{\xi=0} x_{j} y_{k} z_{l} \\
= & -\left(a_{1} b_{1}^{\prime \prime \prime}+3 a_{1}^{\prime} b_{1}^{\prime \prime}+3 a_{1}^{\prime \prime} b_{1}^{\prime}\right) x_{1} y_{1} z_{1}+a_{1} d_{1} f_{2}^{\prime \prime \prime} x_{k+2} y_{k+2} z_{k+2} \\
& +a_{1}^{\prime} d_{1} f_{2}^{\prime \prime} x_{1} y_{k+2} z_{k+2}+a_{1}^{\prime \prime} d_{1} f_{2}^{\prime} x_{1} y_{1} z_{k+2}, \\
C_{2}(x, y, z)= & \left.\sum_{j, k, l}^{n} \frac{\partial^{3} F_{2}(\xi)}{\partial \xi_{j} \partial \xi_{k} \partial \xi_{l}}\right|_{\xi=0} x_{j} y_{k} z_{l} \\
= & -\left(a_{2} b_{1}^{\prime \prime \prime}+3 a_{2}^{\prime} b_{2}^{\prime \prime}+3 a_{2}^{\prime \prime} b_{2}^{\prime}\right) x_{2} y_{2} z_{2}+a_{2} d_{2} f_{1}^{\prime \prime \prime} x_{1} y_{1} z_{1} \\
& +a_{2}^{\prime} d_{1} f_{1}^{\prime \prime} x_{2} y_{1} z_{1}+a_{2}^{\prime \prime} d_{1} f_{1}^{\prime} x_{1} y_{2} z_{2} .
\end{aligned}
$$

When $b=b_{1}$, the restriction of system (2.2) to its two-dimensional center manifold at the critical parameter value can be transformed into the normal form written in complex coordinates [23, 25]:

$$
w \longrightarrow \lambda_{1} w\left(1+\frac{1}{2} d|w|^{2}\right)+O\left(|w|^{4}\right), \quad w \in C
$$


in which

$$
d=\bar{\lambda}_{1}\left\langle p, \widehat{C}(q, q, \bar{q})+2 \widehat{B}\left(q,(1-\widehat{A})^{-1} \widehat{B}(q, \bar{q})\right)+\widehat{B}\left(\bar{q},\left(\lambda_{1}^{2} I-\widehat{A}\right)^{-1} \widehat{B}(q, \bar{q})\right)\right\rangle,
$$

where $p$ and $q$ are defined by (3.1) and $\widehat{B}=\left(B_{1}, B_{2}, 0, \ldots, 0\right)^{T}, \widehat{C}=\left(C_{1}, C_{2}, 0, \ldots, 0\right)^{T}$ with $B_{1}$, $B_{2}, C_{1}$, and $C_{2}$ defined by (3.5).

Theorem 3.1 (see [25]). The direction and stability of the Neimark-Sacker bifurcation are determined by the sign of $\operatorname{Re}(d)$. If $\operatorname{Re}(d)<0$, then the bifurcation is supercritical, that is, the closed invariant curve bifurcating from the origin is asymptotically stable. If $\operatorname{Re}(d)>0$, then the bifurcation is subcritical, that is, the closed invariant curve bifurcating from the origin is unstable.

$$
\text { If } a_{1}\left(x_{1}(n)\right)=1, a_{2}\left(x_{2}(n)\right)=1, b_{1}\left(x_{1}(n)\right)=\tilde{b}_{1} x_{1}(n), b_{2}\left(x_{1}(n)\right)=\tilde{b}_{2} x_{1}(n) \text {, and } f_{1}=f_{2},
$$
system (1.1) reduces to the following discrete time Hopfield neural networks:

$$
\begin{aligned}
& x_{1}(n+1)=\alpha_{1} x_{1}(n)+d_{2} f\left(x_{2}\left(n-k_{2}\right)\right), \\
& x_{2}(n+1)=\alpha_{2} x_{2}(n)+d_{1} f\left(x_{1}\left(n-k_{1}\right)\right),
\end{aligned}
$$

where $\alpha_{1}=1-\widetilde{b}_{1}$ and $\alpha_{2}=1-\widetilde{b}_{2}$.

Corollary 3.2. Suppose system (1.1) satisfies $\left(H_{1}\right)-\left(H_{3}\right)$ and the following condition:

$\left(\mathrm{H}_{4}\right) f^{\prime}(0) \neq 0, f^{\prime \prime}(0)=0$ and $f^{\prime \prime \prime}(0) \neq 0$,

then system (3.8) undergoes a Neimark-Sacker bifurcation from the origin near $b=b_{1}$. If $f^{\prime \prime \prime}(0) / f^{\prime}(0)<0\left(f^{\prime \prime \prime}(0) / f^{\prime}(0)>0\right)$, the Neimark-Sacker bifurcation is supercritical (subcritical) and the bifurcating periodic solutions are asymptotically stable (unstable).

Proof. Since $f^{\prime \prime}(0)=0$, it is easy to know from (3.5) that $B_{1}=0, B_{2}=0$, and

$$
C_{1}(q, q, \bar{q})=a_{1}(0) d_{1} f^{\prime \prime \prime}(0), \quad C_{2}(q, q, \bar{q})=a_{2}(0) d_{2} f^{\prime \prime \prime}(0)\left(\frac{a_{1}(0) d_{1} f^{\prime}(0)}{\lambda_{1}-\alpha_{1}}\right)^{2} \frac{a_{1}(0) d_{1} f^{\prime}(0)}{\bar{\lambda}_{1}-\alpha_{1}} .
$$

Substituting (3.9) into $d$ in (3.6), we can obtain

$$
\operatorname{Re} d=\left|\lambda_{1}\right|^{2}\left[1+\left(\frac{a_{1}(0) d_{1} f^{\prime}(0)}{\left|\lambda_{1}-\alpha_{1}\right|}\right)^{2}\right] \operatorname{Re}\left\{\bar{E}_{1} \frac{f^{\prime \prime \prime}(0)}{f^{\prime}(0)}\right\} .
$$

Note that

$$
\begin{aligned}
\operatorname{sign}\left\{\operatorname{Re} \bar{E}_{1}\right\} & =\operatorname{sign}\left\{\operatorname{Re}\left[k+2+\frac{\alpha_{1}}{\lambda_{1}-\alpha_{1}}+\frac{\alpha_{2}}{\lambda_{1}-\alpha_{2}}\right]\right\} \\
& =\operatorname{sign}\left\{k+\frac{1-\alpha_{1} \cos \theta_{1}}{1+\alpha_{1}^{2}-2 \alpha_{1} \cos \theta_{1}}+\frac{1-\alpha_{2} \cos \theta_{1}}{1+\alpha_{1}^{2}-2 \alpha_{2} \cos \theta_{1}}\right\}=1>0 .
\end{aligned}
$$

Hence, $\operatorname{sign}\{\operatorname{Re} d\}=\operatorname{sign}\left\{f^{\prime \prime \prime}(0) / f^{\prime}(0)\right\}$. Thus the results hold from Theorem 3.1.

Similar results for continuous system corresponding to (3.8) are obtained in [16]. 


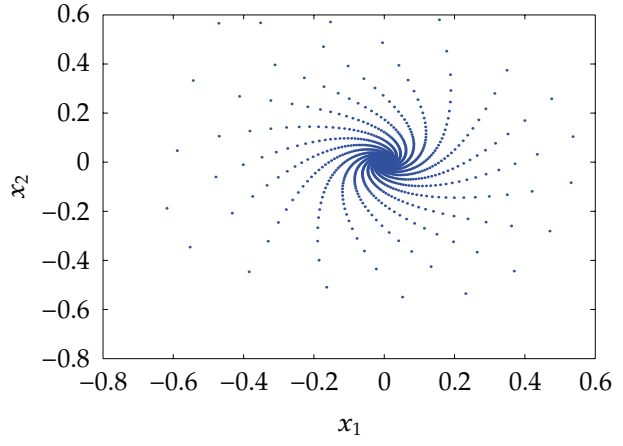

(a)

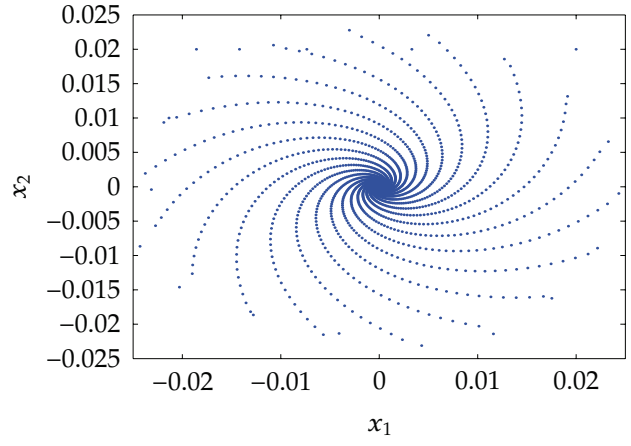

(b)

Figure 1: (a) Phase plot in space $\left(x_{1}, x_{2}\right)$ for system (4.1) with initial condition $(0.02,0.02)$ and $d_{1}=0.806$. (b) Phase plot in space $\left(x_{1}, x_{2}\right)$ for system $(4.1)$ with initial condition $(0.6,0.6)$ and $d_{1}=0.806$.

\section{A Numerical Example}

Consider the following discrete-time Cohen-Grossberg neural network with discrete delays:

$$
\begin{gathered}
x_{1}(n+1)=x_{1}(n)-0.2\left(2+\cos \left(x_{1}(n)\right)\right)\left[0.6 x_{1}(n)-d_{1} \tanh \left(x_{2}(n-3)\right)\right], \\
x_{2}(n+1)=x_{2}(n)-0.2\left(2+\cos \left(x_{2}(n)\right)\right)\left[x_{2}(n)+\sin \left(x_{1}(n-2)\right)\right] .
\end{gathered}
$$

We can obtain that $\theta_{1}=0.3467$ and furthermore we obtain that $b_{1}=-0.2896$ in view of bisection method by using MATLAB. It is easy to know that $b_{0}=0.256$.

According to Theorem 2.1, the null solution of system (4.1) is asymptotically stable when $b \in(-0.2896,0.256)$, and when $b=b_{1}$, the Neimark-Sacker bifurcation occurs at the origin.

Since $f_{2}^{\prime \prime \prime}(0)=-2, f_{1}^{\prime \prime \prime}(0)=-1, f_{2}^{\prime}(0)=1, f_{2}^{\prime}(0)=1, f^{\prime \prime}(0)=0$, and $a_{1}^{\prime}(0)=a_{2}^{\prime}(0)=0$, $B_{1}=B_{2}=0$, sign $\left\{\operatorname{Re} E_{1}\right\}=1>0$, we have

$$
\operatorname{sign}\{\operatorname{Re} d\}=\operatorname{sign}\left\{\operatorname{Re}\left[\frac{f_{2}^{\prime \prime \prime}(0)}{f_{2}^{\prime}(0)}+\frac{f_{1}^{\prime \prime \prime}(0)}{f_{1}^{\prime}(0)}\left(\frac{a_{1}(0) d_{1} f^{\prime}(0)}{\left|\lambda_{1}-\alpha_{1}\right|}\right)^{2}\right]\right\}=-1<0
$$

So the Neimark-Sacker bifurcation is supercritical and the bifurcating periodic solution is stable.

Case 1. Let $d_{1}=0.79$ and $b=-0.2844 \in(-0.2896,0.256)$; then the null solution of system $(4.1)$ is asymptotically stable. Figure 1 shows the dynamic behavior of system (4.1) with initial conditions $(0.02,0.02)$ and $(0.6,0.6)$, respectively. 


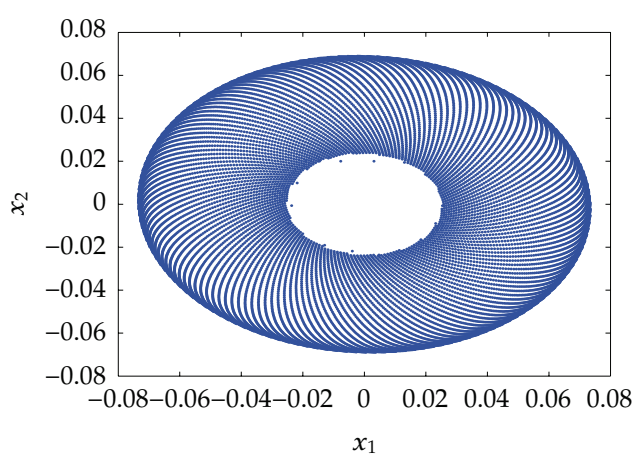

(a)

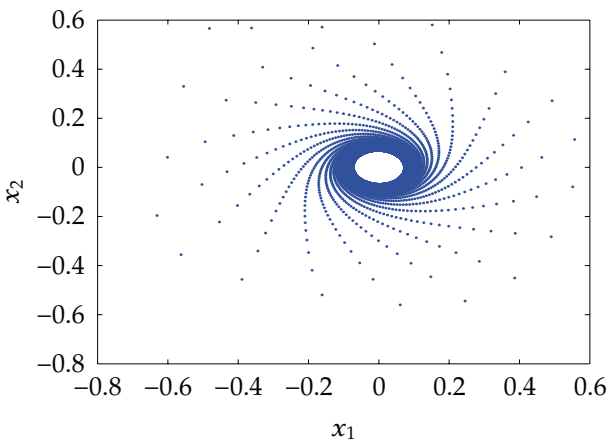

(b)

Figure 2: (a) Phase plot in space $\left(x_{1}, x_{2}\right)$ for system (4.1) with initial condition $(0.02,0.02)$ and $d_{1}=0.85$. (b) Phase plot in space $\left(x_{1}, x_{2}\right)$ for system $(4.1)$ with initial condition $(0.6,0.6)$ and $d_{1}=0.85$.

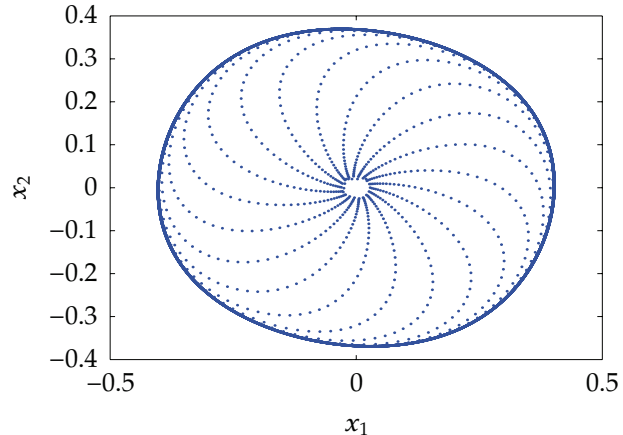

(a)

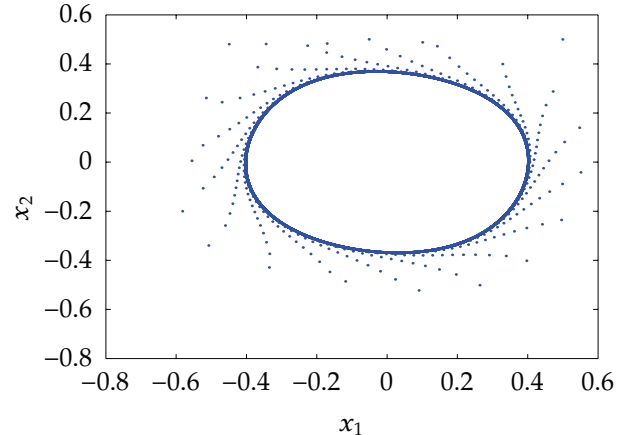

(b)

Figure 3: Bifurcation diagram in $\left(d_{1}, x_{1}\right)$-plane for system (4.1) with step size of 0.01 for $d_{1}$ and initial condition $(0.001,0.001)$, in which there are 100 points for each $d_{1}$.

Case 2. Let $d_{1}=0.806$ and $d_{1}=0.85$, respectively; then $b=-0.2902$ and $b=-0.306$, respectively, and $b<b_{1}=-0.2896$, thus Neimark-Sacker bifurcation occurs at the origin and the bifurcating periodic solutions are stable. Figures 2 and 3 show the dynamic behaviors of system $(4.1)$ with initial conditions $(0.02,0.02)$ and $(0.6,0.6)$, respectively.

Case 3. Let $d_{1}=3.9$ and $d_{1}=-3.4$, respectively; then system (4.1) may be chaostic. Figures 4 and 5 show the dynamic behaviors of system (4.1) with initial condition $(0.02,0.02)$.

\section{Conclusions}

A class of Cohen-Grossberg neural networks with discrete delays is analyzed in this paper. By using $b=a_{1}(0) a_{2}(0) d_{1} d_{2} f_{1}^{\prime}(0) f_{2}^{\prime}(0)$ as a bifurcation parameter, we show that this system undergoes Neimark-Sacker bifurcations at the critical parameter

$$
b=b_{1}=-\sqrt{\left(1+\alpha_{1}^{2}-2 \alpha_{1} \cos \theta_{1}\right)\left(1+\alpha_{2}^{2}-2 \alpha_{2} \cos \theta_{1}\right)},
$$




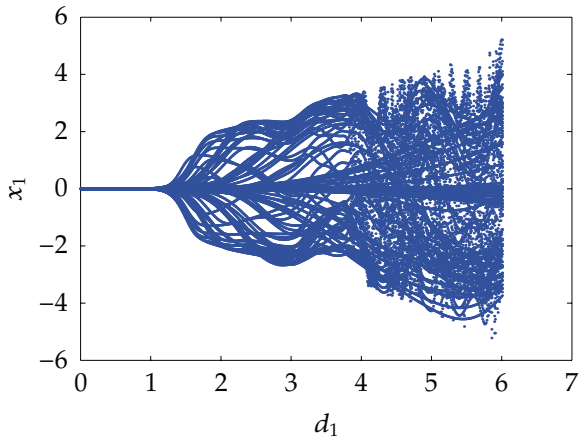

(a)

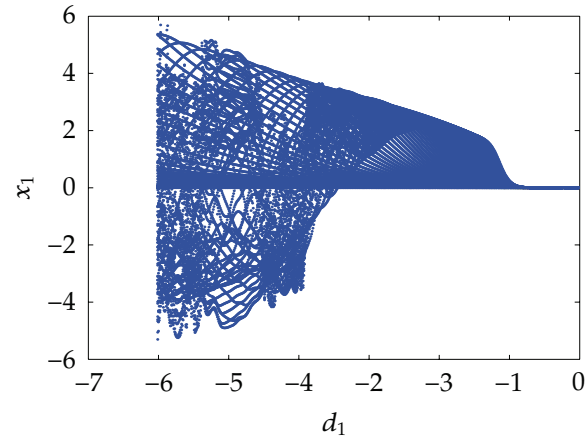

(b)

Figure 4: (a) Phase plot in space $\left(x_{1}, x_{2}\right)$ for system (4.1) with initial condition $(0.6,0.6)$ and $d_{1}=0.79$. (b) Phase plot in space $\left(x_{1}, x_{2}\right)$ for system (4.1) with initial condition $(0.02,0.02)$ and $d_{1}=0.79$.

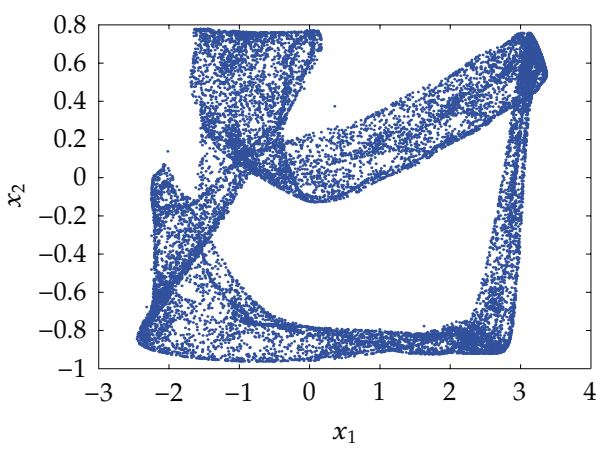

(a)

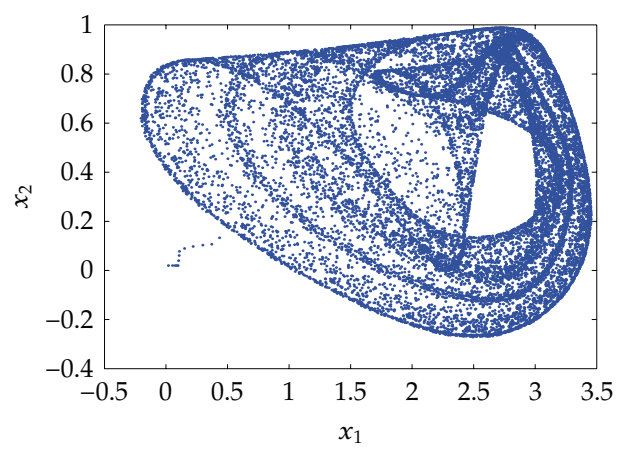

(b)

Figure 5: (a) Phase plot in space $\left(x_{1}, x_{2}\right)$ for system (4.1) with initial condition $(0.02,0.02)$ and $d_{1}=3.9$. (b) Phase plot in space $\left(x_{1}, x_{2}\right)$ for system (4.1) with initial condition $(0.02,0.02)$ and $d_{1}=-3.4$.

where $\theta_{1}$ is the unique solution in $(0, \pi /(k+2))$ of the equation $\sin (k+2) \theta-\left(\alpha_{1}+\alpha_{2}\right) \sin (k+1) \theta+$ $\alpha_{1} \alpha_{2} \sin k \theta=0$ in which $\alpha_{1}=1-a_{1}(0) b_{1}^{\prime}(0)$ and $\alpha_{2}=1-a_{2}(0) b_{2}^{\prime}(0)$. The direction of NeimarkSacker bifurcation and the stability of the bifurcating periodic solutions are investigated by applying the normal form theory and the center manifold theorem for discrete time system. In addition, system may be chaostic when $|b|$ is large enough. The presented numerical simulations demonstrated the theoretical results.

\section{Acknowledgment}

This work was supported by the National Natural Science Foundation of China under Grant no. 11071254 .

\section{References}

[1] J. J. Hopfield, "Neural networks and physical systems with emergent collective computational abilities," Proceedings of the National Academy of Sciences of the United States of America, vol. 79, no. 8, pp. 2554-2558, 1982. 
[2] M. A. Cohen and S. Grossberg, "Absolute stability of global pattern formation and parallel memory storage by competitive neural networks," IEEE Transactions on Systems, Man, and Cybernetics, vol. 13, no. 5, pp. 815-826, 1983.

[3] R. Rakkiyappan, P. Balasubramaniam, and S. Lakshmanan, "Robust stability results for uncertain stochastic neural networks with discrete interval and distributed time-varying delays," Physics Letters A, vol. 372, no. 32, pp. 5290-5298, 2008.

[4] R. Rakkiyappan and P. Balasubramaniam, "Delay-dependent asymptotic stability for stochastic delayed recurrent neural networks with time varying delays," Applied Mathematics and Computation, vol. 198, no. 2, pp. 526-533, 2008.

[5] R. Rakkiyappan and P. Balasubramaniam, "New global exponential stability results for neutral type neural networks with distributed time delays," Neurocomputing, vol. 71, no. 4-6, pp. 1039-1045, 2008.

[6] W. Lu and T. Chen, " $R_{+}^{n}$-global stability of a Cohen-Grossberg neural network system with nonnegative equilibria," Neural Networks, vol. 20, no. 6, pp. 714-722, 2007.

[7] C. Bai, "Stability analysis of Cohen-Grossberg BAM neural networks with delays and impulses," Chaos, Solitons and Fractals, vol. 35, no. 2, pp. 263-267, 2008.

[8] Q. Song and J. Zhang, "Global exponential stability of impulsive Cohen-Grossberg neural network with time-varying delays," Nonlinear Analysis: Real World Applications, vol. 9, no. 2, pp. 500-510, 2008.

[9] R. Rakkiyappan and P. Balasubramaniam, "Dynamic analysis of Markovian jumping impulsive stochastic Cohen-Grossberg neural networks with discrete interval and distributed time-varying delays," Nonlinear Analysis: Hybrid Systems, vol. 3, no. 4, pp. 408-417, 2009.

[10] J. Cao and J. Liang, "Boundedness and stability for Cohen-Grossberg neural network with timevarying delays," Journal of Mathematical Analysis and Applications, vol. 296, no. 2, pp. 665-685, 2004.

[11] J. Cao and X. Li, "Stability in delayed Cohen-Grossberg neural networks: LMI optimization approach," Physica D, vol. 212, no. 1-2, pp. 54-65, 2005.

[12] F. Ren and J. Cao, "Periodic solutions for a class of higher-order Cohen-Grossberg type neural networks with delays," Computers E Mathematics with Applications, vol. 54, no. 6, pp. 826-839, 2007.

[13] X. Yang, "Existence and global exponential stability of periodic solution for Cohen-Grossberg shunting inhibitory cellular neural networks with delays and impulses," Neurocomputing, vol. 72, no. 10-12, pp. 2219-2226, 2009.

[14] W. Xiong and J. Cao, “Global exponential stability of discrete-time Cohen-Grossberg neural networks," Neurocomputing, vol. 64, no. 1-4, pp. 433-446, 2005.

[15] H. Zhao and L. Wang, "Hopf bifurcation in Cohen-Grossberg neural network with distributed delays," Nonlinear Analysis: Real World Applications, vol. 8, no. 1, pp. 73-89, 2007.

[16] J. Wei and S. Ruan, "Stability and bifurcation in a neural network model with two delays," Physica D, vol. 130, no. 3-4, pp. 255-272, 1999.

[17] C. Huang, L. Huang, J. Feng, M. Nai, and Y. He, "Hopf bifurcation analysis of a two-neuron network with four delays," Chaos, Solitons and Fractals, vol. 34, no. 3, pp. 795-812, 2007.

[18] H. Zhao and L. Wang, "Stability and bifurcation for discrete-time Cohen-Grossberg neural network," Applied Mathematics and Computation, vol. 179, no. 2, pp. 787-798, 2006.

[19] E. Kaslik and St. Balint, "Bifurcation analysis for a discrete-time Hopfield neural network of two neurons with two delays and self-connections," Chaos, Solitons and Fractals, vol. 39, no. 1, pp. 83-91, 2009.

[20] E. Kaslik and S. Balint, "Complex and chaotic dynamics in a discrete-time-delayed Hopfield neural network with ring architecture," Neural Networks, vol. 22, no. 10, pp. 1411-1418, 2009.

[21] Q. Gan, R. Xu, W. Hu, and P. Yang, "Bifurcation analysis for a tri-neuron discrete-time BAM neural network with delays," Chaos, Solitons and Fractals, vol. 42, no. 4, pp. 2502-2511, 2009.

[22] S. Guo, X. Tang, and L. Huang, "Stability and bifurcation in a discrete system of two neurons with delays," Nonlinear Analysis: Real World Applications, vol. 9, no. 4, pp. 1323-1335, 2008.

[23] Y. A. Kuznetsov, Elements of Applied Bifurcation Theory, vol. 112 of Applied Mathematical Sciences, Springer, New York, NY, USA, 2nd edition, 1998.

[24] R. S. Varga, Matrix Iterative Analysis, vol. 27 of Springer Series in Computational Mathematics, Springer, Berlin, Germany, 2000.

[25] Yu. A. Kuznetsov and H. G. E. Meijer, "Numerical normal forms for codim 2 bifurcations of fixed points with at most two critical eigenvalues," SIAM Journal on Scientific Computing, vol. 26, no. 6, pp. 1932-1954, 2005. 


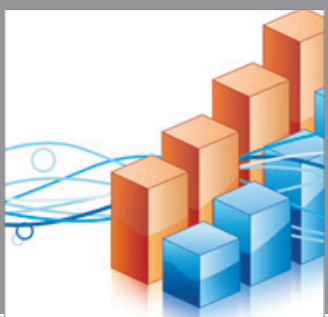

Advances in

Operations Research

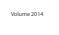

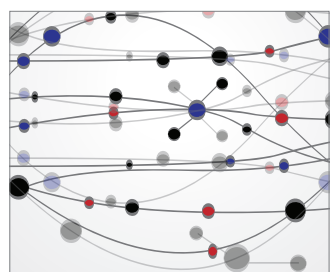

\section{The Scientific} World Journal
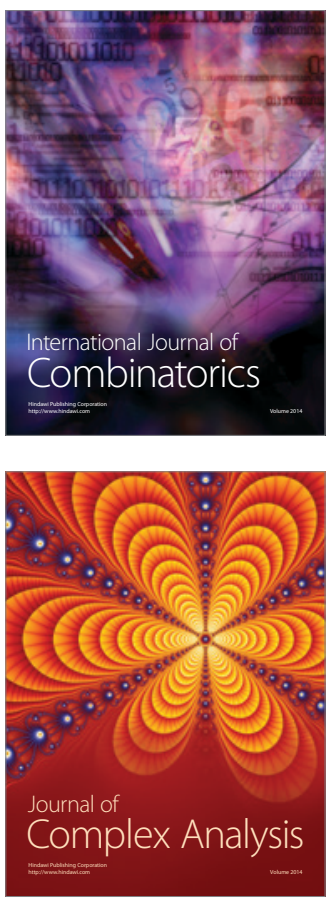

International Journal of

Mathematics and

Mathematical

Sciences
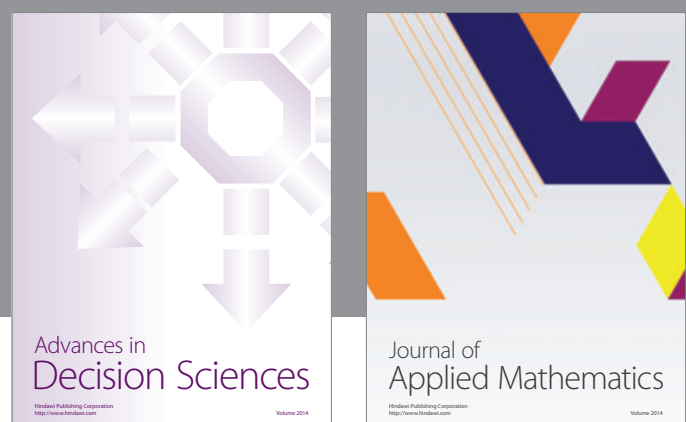

Journal of

Applied Mathematics
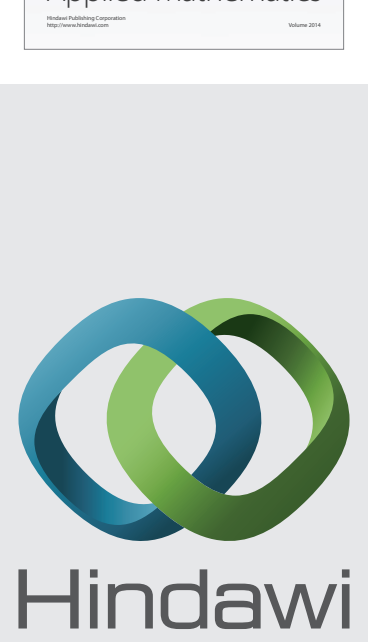

Submit your manuscripts at http://www.hindawi.com
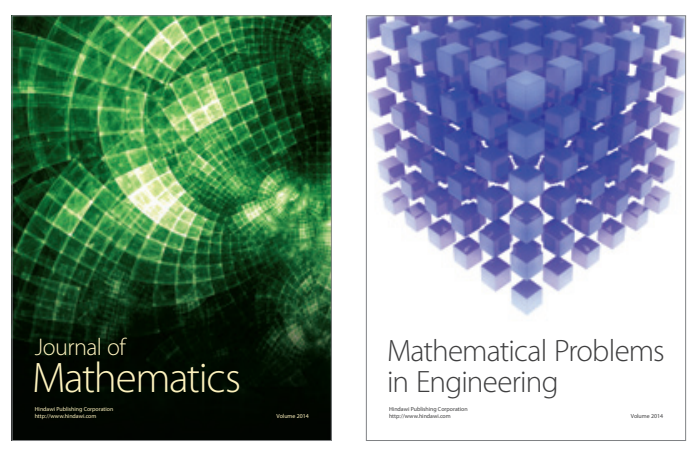

Mathematical Problems in Engineering
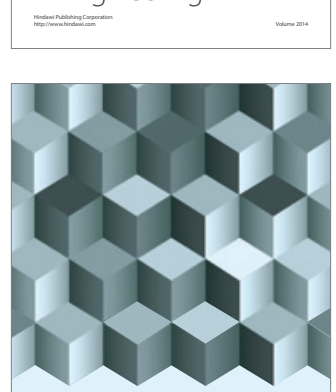

Journal of

Function Spaces
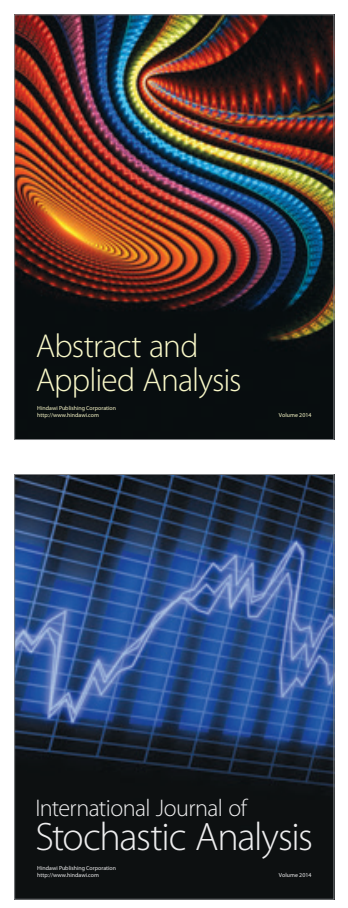

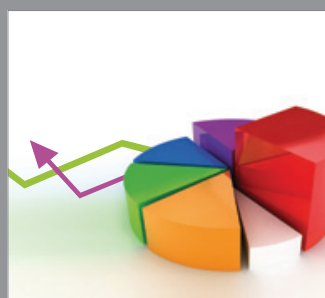

ournal of

Probability and Statistics

Promensencen
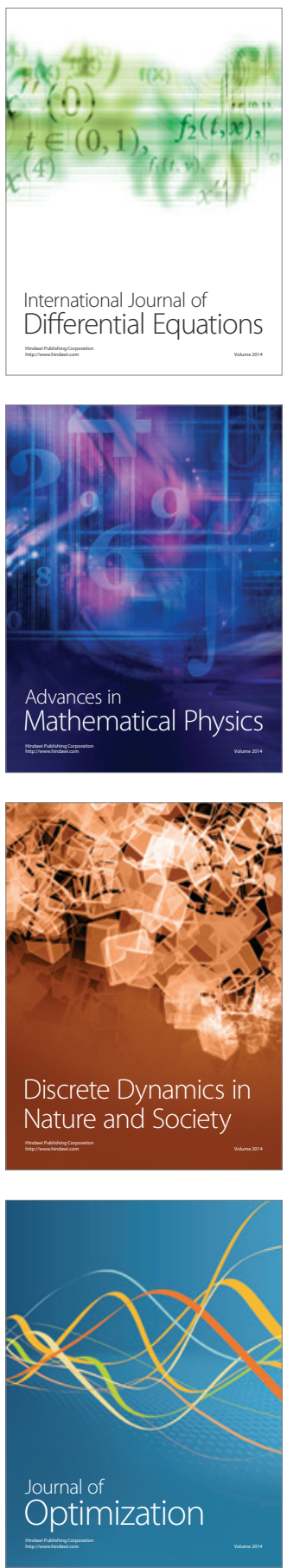